\title{
Munchausen syndrome as scorpion biting: a misdiagnosis
}

\author{
Reza Bidaki ${ }^{1,2}$, Mehrdad Roozbeh ${ }^{3 *}$, Mohamad Amin Sharifi ${ }^{3}$, Seyed Nader Mostafavi ${ }^{4}$, Mohammad Ali \\ Abdolkarimi Davarani ${ }^{5}$
}

\author{
'Department of Psychiatry, Research Center of Addiction and Behavioral Sciences, Shahid Sadoughi University of \\ Medical Sciences, Yazd, Iran \\ ${ }^{2}$ Diabetes Research Center, Shahid Sadoughi University of Medical Sciences, Yazd, Iran \\ ${ }^{3}$ Student Research Committee, Shahid Sadoughi University of Medical Sciences, Yazd, Iran \\ ${ }^{4}$ Department of Forensic Medicine, Shahid Sadoughi University of Medical Sciences, Yazd, Iran \\ ${ }^{5}$ Department of Internal Medicine, Rafsanjan University of Medical Sciences, Rafsanjan, Iran
}

Received: 10 March 2016
Accepted: 29 June 2017
Published online: 1 July 2017
*Corresponding author: Mehrdad
Roozbeh; Email:
mehrdadroozbeh@gmail.com
Competing interests: None.
Funding information: None.
Citation: Bidaki R, Roozbeh M, Sharifi
MA, Mostafavi SN, Davarani MA.
Munchausen syndrome as scorpion
biting: a misdiagnosis. Journal of
Emergency Practice and Trauma
2018; 4(2): $86-88$. doi: $10.15171 /$
jept.2016.15.

Received: 10 March 2016

ccepted: 29 June 2017

*Corresponding author: Mehrdad Roozbeh; Email:

mehrdadroozbeh@gmail.com

Competing interests: None.

Funding information: None. Munchausen syndrome as scorpion

biting: a misdiagnosis. Journal of

\begin{abstract}
Factitious disorder as Munchausen syndrome is a time and money consuming condition that is presented with different signs and symptoms. In emergent situations, it is not a differential diagnosis with high priority, but when it becomes recurrent with atypical symptoms, factitious disorder and malingering should be considered as well. In this survey, we report an odd and interesting presentation of a factitious disease as a scorpion sting which misled some professional healthcare providers until the patient finally confessed that she used turmeric to mimic the signs of a scorpion sting. She exaggerated her symptoms when asked about the details of the mechanism of the scorpion bite. When encountering an emergent condition, after ruling out the critical conditions especially when the symptom is recurrent, factitious disorders should be considered. Consultation with a psychiatrist help the team manage the patient properly.

Keywords: Factitious disorder, Scorpion sting, Turmeric
\end{abstract}

\section{Introduction}

Factitious disorders, including Munchausen syndrome, are one of the most prevalent cases health care providers might encounter anytime (1). Munchausen syndrome patients intentionally mimic their symptoms to be admitted at hospital and gain the attention of the healthcare providers (2). Unfortunately, a significant amount of resources are spent yearly to treat patients with factitious disorders. Factitious disorders include psychological or physical symptoms that are deliberately made by the patient to presume sick role (3). Unlike malingering, in factitious disorders the patient does not make a claim out of personal gain. As their motivation is unknown, personality disorders are suggested as probable cause (4). Logically, for the emergent cases, it is necessary to act as soon as possible and leave the psychological causes for the later assessments. Scorpion envenoming is a common event in developing and underdeveloped countries with the fact that these countries are mainly tropical and subtropical. Scorpion venom mainly affects cardiovascular, respiratory and neural systems (5). There are case reports of "posterior reversible encephalopathy syndrome" (6), Toxic myocarditis (7) following a scorpion sting. As we have dangerous types of scorpions in Iran with serious mortalities and morbidities like coma and necrosis of the tissue (8), we take serious care of patients complaining about scorpion sting. This article presents a woman with Munchausen syndrome who used turmeric to mimic the symptoms of the scorpion sting (Figure 1).

\section{Case presentation}

We intend to present a woman with uncommon and odd presentation of scorpion stings for several times in a short period. A 49-year-old married woman complaining of scorpion stings was referred to the emergency toxicity center. She complained of sudden restlessness, tingling, nausea, vomiting and palpitation. In the physical examination, petechiae and redness were noticed in the left upper quadrant of her left breast. The vital signs and the neurologic examinations were normal (blood pressure: $116 / 61$, pulse rate: 82 , respiratory rate: 18 , temperature: 37.5, Glasgow coma scale [GCS]: 15/15). In the past medical history there were three documented times of hospitalization in the last year with the same 


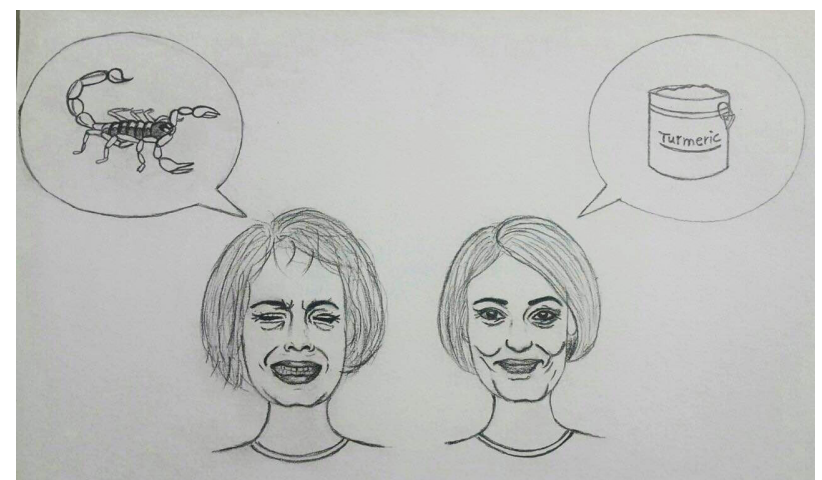

Figure 1. A woman that induces a factitious disorder for achievement of primary gain. She has preoccupation with damage to skin. She indicate that it is bitten by the scorpion sting.

chief complaint as being stung by the scorpion. She had also been hospitalized in intensive care unit (ICU). She mentioned using dexamethasone and metoclopramide for her allergy in the last year. Complete blood count, creatinine, liver function tests, creatine phosphokinase, lactate dehydrogenase and urine analysis were checked for the patient and she was advised to be NPO. Intravenous crystalloids were started for the patient. As gross hematuria was detected in her urine analysis, the patient had a vial of scorpion anti-venom. After being under close monitoring, she became stable and was discharged from the hospital 2 days later.

Next year she referred to the emergency room, complaining about scorpion stings in her right thigh and both lower extremities. She had generalized paresthesia, headache, nausea and vomiting and local itching at the site of stings. The physical examinations and vital signs were normal. Cimetidine, hydrocortisone and antivenom were administered. After administering the drugs, she had respiratory distress and palpitation, emergent electrocardiography (ECG) was done and the results were normal. As the patient was still unstable she was transferred to ICU for accurate monitoring in ICU. She was found depressed as she did not respond to the verbal communication. So, the psychiatric consultation was requested but unfortunately she left the hospital before the psychiatrist could visit her. One month later, she referred to the hospital complaining about shortness of breath and scorpion sting at the posterior region of the left leg. Likewise the previous times, she had paresthesia and itching but the physical examinations were normal. The history obtained from her family revealed that she had frequently used variety of benzodiazepines and phenothiazines. As the patient was seemingly icteric, the liver function tests were requested. The laboratory tests were normal. In the follow-up, she was still complaining about scorpion stings. She even provided the scorpion in her following referrals but her family did not allow further test and discharged her. The patient was referred to a dermatologist for her itchy lesion. After skin biopsy, she was diagnosed with "lichen planus". As the disease became generalized, she used systemic corticosteroid.
The psychiatric consultation was requested, she did not have any history of suicide, but she had severe anxiety and mild depression. She was raised in a chaotic family that exaggerated her symptoms and when asked about the details of the mechanism of the scorpion bite, she confabulated. Fortunately, the toxicology specialist noticed that the yellow color on skin was removable by the alcohol. All the previous physicians could not figure out the real problem. She confessed that she was not satisfied from her husband and her quality of life. So she had fabricated the sign and symptoms of the scorpion sting to get more attention. The psychiatrist confirmed she played a sick role, had attention seeking behavior and got pleasure from it. Finally, when her motivations were revealed she did not come back to the hospital again.

\section{Discussion}

Scorpion sting is an emergent condition especially in the countries with dangerous species of scorpion. In some cases with recurrent atypical symptoms and without any clue for confirmation of the scorpion sting, a factitious disease should be considered. Factitious disorder (especially Munchausen syndrome) is more common but not restricted to the nurses and healthcare workers (9). Taking a detailed history can help the specialist to better diagnose the condition. The patient did not have a proper emotional feedback as well as personal satisfaction. When somebody is unable to express his/her sufferings, he/ she fabricates groups of somatic diseases intentionally resulting in misleading the healthcare providers. It should be noted that unlike malingering, in factitious disorders the patient does not gain financial benefits. A detailed history and assessing the patient's motivation can help to diagnose these disorders. Looking for the presence of anxiety, depression, unusual behavior, recent or childhood traumatic experiences can help identify psychiatric causes of the factitious disorders.

\section{Conclusion}

When encountering an emergent condition, after ruling out the critical conditions especially when the symptom is recurrent, factitious disorders should be considered. Consultation with a psychiatrist help the team manage the patient safely and properly.

\section{Ethical issues}

The authors considered all subjects related ethical issue and the patient consent was taken.

\section{Authors' contributions}

SNM visited the patient in toxicology ward. RB conceived the psychiatric evaluation and collected the clinical data.MR searched the Medline, wrote and clarified the manuscript. $\mathrm{RB}$ and $\mathrm{MR}$ revised and edited the manuscript. MASand MAD prepared the manuscript and illustrated the case. All authors read and approved the final manuscript. 


\section{References}

1. Folks DG. Munchausen's syndrome and other factitious disorders. Neurol Clin 1995; 13(2): 267-81.

2. Doherty AM, Sheehan JD. Munchausen's syndrome--more common than we realize? Ir Med J 2010; 103(6): 179-81.

3. Feldman M. The costs of factitious disorders. Psychosomatics 1994; 35(5): 506-7.

4. Clarke E, Melnick SC. The Munchausen syndrome or the problem of hospital hoboes. Am J Med 1958; 25(1): 6-12. doi: 10.1016/0002-9343(58)90192-X.

5. Ismail $\mathrm{M}$. The scorpion envenoming syndrome. Toxicon 1995; 33(7): 825-58.

6. Porcello Marrone LC, Marrone BF, Neto FK, Costa FC, Thomé GG, Aramburu MB, et al. Posterior reversible encephalopathy syndrome following a scorpion sting. J Neuroimaging 2013; 23(4): 535-6. doi: 10.1111/jon.12017.

7. Karakurt C, Koçak G. Toxic myocarditis after scorpion envenomation: case report. J Turgut Ozal Med Cent 2007; 14(1): 61-3. [In Turkish].

8. Dehghani R, Fathi B. Scorpion sting in Iran: a review. Toxicon 2012; 60(5): 919-33. doi: 10.1016/j.toxicon.2012.06.002.

9. Wallach J. Laboratory diagnosis of factitious disorders. Arch Intern Med 1994;154(15):1690-6. doi: 10.1001/ archinte.1994.00420150048005. 